\title{
Enunciados diagnósticos enfermeros más frecuentes, que cumplen con la taxonomía II NANDA registrado en el servicio de medicina del Hospital Nacional Arzobispo Loayza
}

\author{
Peralta-Ugarte Julio ${ }^{1}$, Rafael-Romero Mirtha ${ }^{1}$, Saravia-Neira Lizbbet ${ }^{1}$, Mayorca-Carmelo Carmen ${ }^{2}$
}

\section{RESUMEN}

Objetivo: identificar los enunciados diagnósticos enfermeros más frecuentes, que cumplen los criterios de la taxonomía II de North American Nursing Diagnosis Association (NANDA), registrados en las notas de enfermería por las enfermeras del Servicio de Medicina 4I del Hospital Nacional Arzobispo Loayza, de Lima, Perú. Material y métodos: estudio descriptivo, observacional y transversal. La muestra estuvo conformada por 424 diagnósticos de enfermería realizados durante los meses de julio a septiembre de 2011. Para la recolección de datos el instrumento usado fue una ficha de diagnóstico enfermero elaborada por los autores; para la confiabilidad se realizó una prueba piloto. Resultados: el 73,6\% de diagnósticos que cumplían con la etiqueta según la taxonomía II de NANDA; el 34,7\% de diagnósticos con las características definitorias; el 44,1\% de diagnósticos con los factores relacionados o de riesgo, y el 33,7\% de diagnósticos que cumplen con las tres dimensiones. Se identificó doce diagnósticos reales, de los cuales los más frecuentes fueron: 82 diagnósticos de dolor agudo relacionado con agente lesivo biológico 19,3\%; 16 diagnósticos de ansiedad relacionado con cambios en el estado de salud 3,8\%, y otros. Conclusiones: se identificó que del total de diagnósticos enfermeros evaluados y registrados se aprobaron solo 143 (33,7\%) porque cumplían con las tres dimensiones según la taxonomía II de NANDA, siendo la más frecuente dolor agudo relacionado con agente lesivo biológico debido a que los pacientes padecen enfermedades crónicas; se evidencia el dolor como un síntoma común a lo largo del proceso de su enfermedad.

Palabras clave: taxonomía II de NANDA - I, diagnósticos enfermeros, historias clínicas. Perú. (Fuente DeCs BIREME).

\section{The most frequent nursing diagnoses statements that meet the criteria of the NANDA taxonomy II recorded in the medicine department at the Hospital Nacional Arzobispo Loayza}

\begin{abstract}
Objetive: To identify the most frequent nursing diagnoses statements that meet the criteria of the NANDA Taxonomy II recorded in the nursing notes by nurses of 4 I medicine department at the Hospital Nacional Arzobispo Loayza LimaPerú. Material and Methods: This is a descriptive, observational and transversal study. The sample consisted of 424 nursing diagnoses made during the months of July to September 2011. For data collection instrument used was a nursing diagnosis form developed by the authors, for reliability was piloted. Results: It was found $73.6 \%$ who met with the label according to NANDA Taxonomy II, $34.7 \%$ with the defining characteristics, $44.1 \%$ related factors or risk and $33.7 \%$ that meet the 3 dimensions identifying 12 real diagnoses of which the most frequent are: 82 diagnoses of acute pain related biological offending agent 19.3\%, 16 diagnoses of anxiety related changes in health status $3.8 \%$. Conclusion:It was identified that of the total registered diagnoses were approved only 143 diagnoses (33.7\%) because they met the 3 dimensions of the NANDA taxonomy II. being the most frequent acute pain related biological agent, because patients with chronic diseases, evident pain as a common symptom during the disease process.
\end{abstract}

Key words: NANDA I Taxonomy II, nursing diagnoses, medical histories. Peru. (Source DeCs BIREME).

Alumno de la Facultad de Enfermería. Universidad Peruana Cayetano Heredia. Lima-Perú

Licenciada en Enfermería. Docente de la Facultad de Enfermería de la Universidad Peruana Cayetano Heredia. 


\section{INTRODUCCIÓN}

Enfermería es una profesión sumamente compleja, ya que incluye conocimientos científicos y técnicos; capacidad de comunicación; complicidad emocional, y otras muchas cualidades. Por ello se considera también como un completo proceso de pensamiento que lleva del conocimiento a la técnica, de la percepción a la acción, de la decisión al tacto y de la observación al diagnóstico. Los diagnósticos enfermeros constituyen un marco útil para la investigación del profesional de Enfermería, porque proporcionan mecanismos únicos para estructurar los conocimientos en Enfermería, en un intento de definir su rol y ámbito particular (1). Para llegar a la identificación de los diagnósticos de enfermería se utiliza el proceso de enfermería, el cual consta de cinco fases: valoración, diagnóstico de enfermería, planificación, ejecución y evaluación. Obtuvo legitimidad en 1973 cuando la American Nurse Association (ANA) publicó estándares del ejercicio de la Enfermería, la cual describe estos cinco pasos del proceso. Asimismo, la incorporación en Colombia de los diagnósticos de la taxonomía II de NANDA, ha tenido una amplia difusión en el ámbito educativo, pero han encontrado vacíos en su aplicación en la práctica, especialmente por considerarse que han sido generados en otros contextos internacionales $(2,14)$.

El proceso de enfermería es un método sistemático para brindar cuidados humanistas, y se caracteriza por ser planeado, centrado en el paciente, orientado a problemas, y dirigido a metas; lo que se ve reflejado en las anotaciones de enfermería (3). En la actualidad, la enfermera utiliza el proceso de enfermería para identificar y sintetizar los datos clínicos, y para disponer intervenciones de enfermería que reduzcan, eliminen o prevengan las alteraciones de salud que pertenezcan al dominio legal y educativo de la enfermera $(4,15-16)$. Históricamente las anotaciones de enfermería sobre los cuidados de los pacientes en los hospitales del Perú se han limitado a una narración cronológica de las observaciones más o menos casuales acerca del paciente, efectos del medicamento, tratamiento, datos de monitorización, entre otros. En Enfermería las aplicaciones de los diagnósticos enfermeros tuvieron sus comienzos en la década de 1950 de manera lenta; su origen se ubica en Estados Unidos de América y progresivamente fue extendiéndose al resto del mundo (11).

El pabellón que inició la aplicación de diagnósticos NANDA fue el Servicio de Medicina 4I del Hospital Nacional Arzobispo Loayza, de Lima-Perú en 1997, mediante un formato de anotaciones de enfermería estructurado por cinco partes: datos subjetivos, datos objetivos, diag- nósticos de enfermería, objetivos, plan e intervención y evaluación, para optimizar el cuidado enfermero. En la actualidad, las enfermeras recolectan los datos mediante la valoración de respuestas humanas registradas en las notas de enfermería; registran, además, valores de exámenes de laboratorio (hemograma, urea, creatinina, gases arteriales, entre otros) además de resultados de procedimientos invasivos y no invasivos (aspirado de medula ósea, paracentesis, ecografía renal y ECG) de este modo sustentan el diagnóstico enfermero. Los pacientes hospitalizados en el Servicio de Medicina 4I en el Hospital Nacional Arzobispo Loayza, de Lima-Perú; son personas con enfermedades crónicas degenerativas como diabetes mellitus, insuficiencia renal, insuficiencia respiratoria, entre otros. Asimismo, este servicio es considerado un servicio de contingencia. En el año 2001 atendió pacientes provenientes del incendio ocurrido en el mercado Mesa Redonda; pacientes politraumatizados producto del terremoto del Perú en el 2007; y, por último, pacientes con influenza A(H1N1) en el 2009.

La presente investigación contribuyó a fortalecer y desarrollar un lenguaje estandarizado de la práctica diagnóstica mediante la identificación de las debilidades que se evidencian en la identificación de los diagnósticos NANDA, dando un mayor contenido científico al trabajo de enfermería y al desarrollo de la profesión de acuerdo con la priorización de los diagnósticos NANDA.

\section{MATERIAL Y MÉTODOS}

Estudio descriptivo, observacional y transversal realizado en el Servicio de Medicina 4I del Hospital Nacional Arzobispo Loayza, nivel III, provincia y departamento de Lima, Perú. El pabellón cuenta con diez enfermeras/os asistenciales y diez técnicas de enfermería; se cuenta con una capacidad de 26 camas. La población estuvo constituida por todos los diagnósticos de enfermería descritos en los registros de enfermería de cada historia clínica durante 24 horas en los meses de julio a septiembre de 2011. Se consideraron como criterios de inclusión los diagnósticos enfermeros registrados en las notas de enfermería que consignaran la fecha, el turno, la firma, el sello y el número de colegiatura de las enfermeras que se registraron en el Servicio de Medicina 4I en cada turno, durante las 24 horas de los meses de julio a septiembre de 2011. Asimismo los diagnósticos enfermeros con etiqueta según taxonomía II de NANDA que se identificaron durante estos meses. Se excluyeron aquellos diagnósticos de enfermería que se encontraron registrados en las notas de enfermería de pacientes que fueron dados de alta; los diagnósticos que fueron registrados por estudiantes o in- 
ternos de enfermería, y los diagnósticos de enfermeros de otros servicios que estuvieron de apoyo durante el turno de los meses de julio a septiembre de 2011. Se evaluó un total de 424 diagnósticos de enfermería.

Las definiciones operacionales fueron: etiqueta diagnóstica; denominada así al enunciado que describe el problema de salud con la respuesta real, potencial y de riesgo del paciente. Estas etiquetas están planteadas formalmente por la NANDA y son registradas por la enfermera en las notas de enfermería. Las características definitorias; son denominadas a las claves o interferencias observables que se agrupan como manifestaciones reales de un diagnostico real, de salud o de promoción de la salud. Los factores relacionados; son las causas u origen probable del problema; puede ser determinante, es decir, directo o indirecto, que han contribuido al cambio del estado de salud, registrado en la nota de enfermería. Los factores de riesgo; son los factores ambientales y elementos fisiológicos, psicológicos, genéticos o químicos que incrementan la vulnerabilidad de un individuo, la familia o la comunidad ante un evento no saludable.

Se utilizó como instrumento una lista de chequeo que está formada por un encabezado y nueve ítems. El encabezado recogió información sobre el servicio, la fecha, el número de historia clínica, el nombre del evaluador, y el número de diagnósticos elaborados por cada turno del Servicio de Medicina 4I durante tres días a la semana. La lista de chequeo permitió identificar cuantos enunciados diagnósticos enfermeros cumplen con los criterios de la taxonomía II de NANDA; cada pregunta presentaba dos alternativas: Sí y No. Para la validación del instrumento se sometió a la validez de criterio, contenido y constructo de diez especialistas en el área. La prueba que se utilizó para evaluar los resultados del juicio de expertos fue la binomial, siendo el valor encontrado de $p<0,05$. Por otro lado, se realizó la prueba piloto en una población similar, la cual estuvo conformada por 150 diagnósticos de enfermería del Servicio de Medicina 4 II del Hospital Nacional Arzobispo Loayza, de Lima, Perú. Dicho servicio cuenta con veinticuatro camas y once enfermeras. Los investigadores ingresaron a las $7.30 \mathrm{~h}$ para ser partícipes del reporte de enfermería. Posteriormente, se recolecto ocho historias clínicas por investigador y se observó las notas de enfermería; se identificó y registró en la ficha de chequeo los diagnósticos enfermeros de cada turno. Los investigadores tuvieron en cuenta los criterios de inclusión y exclusión mediante la recolección de los diagnósticos enfermeros elaborados por las enfermeras, lo que permitió obtener la confiabilidad del instrumento determinado por la fórmula 20 de Kuder-Richardson, siendo este 0,70 .
Al finalizar la prueba piloto se realizó los trámites respectivos en la Facultad de Enfermería de la Universidad Peruana Cayetano Heredia, la cual autorizó la aplicación del trabajo de investigación después de ser aprobado el proyecto por el comité de ética. Asimismo, se solicitó permiso a la directora del Hospital Nacional Arzobispo Loayza y al jefe del servicio. Además, se coordinó con la jefa de enfermería para la ejecución del estudio en el servicio de medicina $4 \mathrm{I}$, que cuenta con veintiséis camas y diez enfermeras. Los investigadores que recolectaron los datos del estudio contaron con los recursos y conocimientos adecuados para el llenado del cuestionario el cual se hizo de julio a septiembre de 2011. Se asistió desde las $7.30 \mathrm{~h}$ formando parte del reporte de enfermería del servicio; es decir, se incorporaron al trabajo de enfermería apoyando en la parte asistencial y, por otro lado, para la recolección de los datos por ocho días: lunes 11 , miércoles 20 , y viernes 29 de julio; lunes 8 , miércoles 17 , y viernes 26 de agosto; y lunes 5 y miércoles 14 de septiembre. Se identificaron 424 diagnósticos cuya totalidad se seleccionó para fines del estudio.

Una vez recolectada la información los diagnósticos fueron codificados ordenados y agrupados en la hoja de cálculo del programa Microsoft Office Excel versión 2007. Luego se utilizó el programa estadístico Stata versión 10, para determinar las frecuencias relativas simples según los objetivos de la investigación. Los resultados se presentan en tablas de doble entrada incluyendo frecuencias simples y relativas.

\section{RESULTADOS}

La Tabla 1 muestra que los enunciados diagnósticos enfermeros más frecuentes cumplen con la etiqueta según la taxonomía II de NANDA y alcanzan el mayor porcentaje (73,6\%); el menor porcentaje lo alcanzan los que cumplen con las tres dimensiones según la misma taxonomía $(33,7 \%)$.

La Tabla 2 muestra que el total de enunciados diagnósticos enfermeros más frecuentes, que cumplen con la etiqueta según la Taxonomía II de NANDA llegan al 73,6\%. De los cuales, el más frecuente es el dolor agudo r/c agente lesivo biológico con el 17,7\%; y el menos frecuente es el patrón respiratorio ineficaz $\mathrm{r} / \mathrm{c}$ cambios en la membrana alveolo capilar con el $0,7 \%$.

La Tabla 3 muestra que el total de enunciados diagnósticos enfermeros más frecuentes que cumplen con las características definitorias según la Taxonomía II de 
Tabla 1. Enunciados diagnósticos enfermeros más frecuentes que cumplen con etiqueta, características definitorias y factor relacionado según la taxonomía II de NANDA del Servicio de Medicina 4 I del Hospital Nacional Arzobispo Loayza. Julio a septiembre 2011

\begin{tabular}{|c|c|c|c|c|c|c|}
\hline \multirow{2}{*}{ Diagnósticos de enfermería } & \multicolumn{2}{|c|}{ Sí cumple } & \multicolumn{2}{|c|}{ No cumple } & \multicolumn{2}{|c|}{ Total } \\
\hline & $\mathbf{N}$ & $\%$ & $\mathbf{N}$ & $\%$ & $\mathbf{N}$ & $\%$ \\
\hline Con etiqueta NANDA & 312 & 73,6 & 112 & 26,4 & 424 & 100 \\
\hline Con características definitorias & 147 & 34,7 & 277 & 65,3 & 424 & 100 \\
\hline Con factor relacionado y factor de riesgo & 187 & 44,1 & 237 & 55,9 & 424 & 100 \\
\hline Con las tres dimensiones & 143 & 33,7 & 281 & 66,3 & 424 & 100 \\
\hline
\end{tabular}

Tabla 2. Enunciados diagnósticos enfermeros más frecuentes que cumplen con la etiqueta según la taxonomía II de NANDA del Servicio de Medicina 4 I del Hospital Nacional Arzobispo Loayza. Julio a septiembre 2011

\begin{tabular}{clrr}
\hline $\mathbf{N}^{\circ}$ & \multicolumn{1}{c}{ DIAGNÓSTICOS CON ETIQUETA } & N & $\%$ \\
\hline 1 & Dolor agudo r/c agente lesivo biológico & 75 & 17,7 \\
2 & Ansiedad r/c estancia hospitalaria & 29 & 6,8 \\
3 & Hipertermia r/c proceso infeccio so & 25 & 5,9 \\
4 & Ansiedad r/c cambios en el estado de salud & 21 & 5,0 \\
5 & Riesgo de infección r/c procedimientos invasivos & 19 & 4,5 \\
6 & Deterioro de la integridad cutánea r/c inmovilidad & 19 & 4,5 \\
7 & Limpieza ineficaz de las vías aéreas r/c acumulo de secreciones & 15 & 3,5 \\
8 & Deterioro de la movilidad física r/c debilidad muscular & 9 & 2,1 \\
9 & Riesgo de deterioro de la integridad cutánea r/c inmovilidad & 9 & 2,1 \\
10 & Protección ineficaz r/c perfiles hematológicos disminuidos & 8 & 1,9 \\
11 & Intolerancia a la actividad r/c debilidad muscular & 7 & 1,7 \\
12 & Deterioro del intercambio gaseoso r/c cambio en la membrana alveolo capilar & 5 & 1,2 \\
13 & Déficit del autocuidado: baño, higiene r/c deterioro muscular & 5 & 1,2 \\
14 & Exceso de volumen de líquido r/c compromiso de los mecanismo reguladores & 5 & 1,2 \\
15 & Exceso de volumen de líquido r/c alteración de los mecanismos reguladores & 5 & 1,2 \\
16 & Intolerancia a la actividad r/c fatiga. & 4 & 0,9 \\
17 & Náuseas r/c irritación gástrico & 4 & 0,9 \\
18 & Patrón respiratorio ineficaz r/c cambios en la membrana alveolo capilar & 3 & 0,7 \\
19 & Otros diagnó sticos menos frecuentes & 45 & 10,6 \\
Total de diagnósticos que cumplen & 312 & 73,6 \\
\hline
\end{tabular}


Tabla 3. Enunciados diagnósticos enfermeros más frecuentes que cumplen con las características definitorias según la taxonomía II de NANDA del Servicio de Medicina 4 I del Hospital Nacional Arzobispo Loayza.

Julio a septiembre 2011

\begin{tabular}{|c|c|c|c|c|}
\hline \multirow{2}{*}{$\mathbf{N}^{\circ}$} & \multirow{2}{*}{ DIAGNÓSTICOS CON ETIQUETA } & \multirow{2}{*}{ CARACTERISTICAS DEF INITORIAS } & \multicolumn{2}{|c|}{ TOTAL } \\
\hline & & & $\mathbf{N}$ & $\%$ \\
\hline 1 & Dolor agudo r/c agente lesivo biológico & $\begin{array}{l}\text { - Dolor torácico. } \\
\text { - Tipo sordo. } \\
\text { - Escala del dolor } 3 \text { o } 4\end{array}$ & 65 & 15,3 \\
\hline 2 & Ansiedad $\mathrm{r} / \mathrm{c}$ cambios en el estado de salud & $\begin{array}{l}\text { - Nerviosismo. } \\
\text { - Preocupación } \\
\text { - Movimientos extraños. }\end{array}$ & 10 & 2,4 \\
\hline 3 & Riesgo de infección r/c procedimientos invasivos & $\begin{array}{l}\text { - Estancia prolongada de vía periférica. } \\
\text { - Estancia prolongada de SNG. } \\
\text { - Cambio de pH de las secreciones. }\end{array}$ & 10 & 2,4 \\
\hline 4 & Hipertermia $\mathrm{r} / \mathrm{c}$ proceso infeccioso & $\begin{array}{l}\text { - Calor al tacto. } \\
\text { - Piel enrojecida. } \\
\text { - Taquipnea }\end{array}$ & 9 & 2.1 \\
\hline 5 & $\begin{array}{l}\text { Limpieza ineficaz de las vías aéreas } r / c \text { acumulo } \\
\text { de secreciones }\end{array}$ & $\begin{array}{l}\text { - Tos inefectiva. } \\
\text { - Excesiva cantidad de esputo. } \\
\text { - Agitación. }\end{array}$ & 9 & 2,1 \\
\hline 6 & $\begin{array}{l}\text { Protección ineficaz r/c perfiles hematológicos } \\
\text { disminuidos }\end{array}$ & $\begin{array}{l}\text { - Alteración de la coagulación. } \\
\text { - Deterioro de la cicatrización. } \\
\text { - Disnea. }\end{array}$ & 7 & 1,7 \\
\hline 8 & $\begin{array}{l}\text { Deterioro de la movilidad física r/c debilidad } \\
\text { muscular }\end{array}$ & $\begin{array}{l}\text { - Limitación de la amplitud de los movimien tos. } \\
\text { - In estabilidad postural. } \\
\text { - Movimientos descoordinados. }\end{array}$ & 5 & 1,2 \\
\hline 9 & $\begin{array}{l}\text { Deterioro de la integridad cutánea r/c } \\
\text { inmovilidad }\end{array}$ & $\begin{array}{l}\text { - Destrucción de las capas de la piel. } \\
\text { - Alteración de la superficie de la piel. } \\
\text { - Invasión de las estructuras corporales. }\end{array}$ & 5 & 1,2 \\
\hline 10 & Intolerancia a la actividad $\mathrm{r} / \mathrm{c}$ debilidad muscular & $\begin{array}{l}\text { - Malestar debido al esfuerzo. } \\
\text { - In forme verbal de fatiga. } \\
\text { - Frecuencia card iaca normal en respuesta a la } \\
\text { actividad. }\end{array}$ & 4 & 0,9 \\
\hline 11 & $\begin{array}{l}\text { Déficit de autocuidado: baño, vestido r/c deterioro } \\
\text { muscular }\end{array}$ & $\begin{array}{l}\text { - Incapacidad de llevar los alimentos de los } \\
\text { recipientes a la boca. } \\
\text { - In capacidad para masticar la comida. } \\
\text { - Incapacidad para coger los alimentos con los } \\
\text { utensilios. }\end{array}$ & 3 & 0,7 \\
\hline 12 & $\begin{array}{l}\text { Exceso de volumen de líquido } \mathrm{r} / \mathrm{c} \text { compromiso de } \\
\text { mecan is mos reguladores }\end{array}$ & $\begin{array}{l}\text { - Alteración de electrolitos. } \\
\text { - Cambios de la presión arterial. } \\
\text { - Edema. }\end{array}$ & 2 & 0,5 \\
\hline 13 & $\begin{array}{l}\text { Patrón respiratorio inefectivo r/c cambios en la } \\
\text { membrana alveo lo capilar }\end{array}$ & $\begin{array}{l}\text { - Aleteo nasal. } \\
\text { - Alteración de los movimientos torácicos. } \\
\text { - Taquipnea }\end{array}$ & 2 & 0,5 \\
\hline \multirow[t]{2}{*}{14} & Otros diagnósticos menos frecuentes & & 16 & 3,8 \\
\hline & Total de diagnósticos que cumplen & & 147 & 34,7 \\
\hline
\end{tabular}

NANDA, es de $34,7 \%$. De los cuales, el más frecuente es el dolor agudo $\mathrm{r} / \mathrm{c}$ agente lesivo biológico con un $15,3 \%$, así como los menos frecuentes son el exceso de volumen de líquidos $\mathrm{r} / \mathrm{c}$ compromiso de mecanismos reguladores $\mathrm{y}$ el patrón respiratorio ineficaz $\mathrm{r} / \mathrm{c}$ cambios en la membra- na alveolo capilar con un $0,5 \%$.

La Tabla 4 muestra que el total de enunciados diagnósticos enfermeros más frecuentes que cumplen con el factor relacionado y de riesgo según la Taxonomía II de 
Tabla 4. Enunciados diagnósticos enfermeros más frecuentes que cumplen con factor relacionado y de riesgo según la taxonomía II de NANDA del Servicio de Medicina 4 I del Hospital Nacional Arzobispo Loayza. Julio a septiembre 2011

\begin{tabular}{|c|c|c|c|}
\hline & DIAGNOSTICOS CON FACTOR RELACIONADO Y DE RIESGO & $\mathbf{N}$ & $\%$ \\
\hline \multicolumn{4}{|c|}{ Diagnósticos con factor relacionado } \\
\hline 1 & Dolor agudo r/c agente lesivo bioló gico & 67 & 15,8 \\
\hline 2 & Limpieza in eficaz de las vías aéreas $\mathrm{r} / \mathrm{c}$ acú mulo de secrecio nes & 14 & 3,3 \\
\hline 3 & Ansiedad r/c cambios en el estado de salud & 13 & 3,1 \\
\hline 4 & Deterioro de la integridad cutánea $\mathrm{r} / \mathrm{c}$ inmovilidad & 10 & 2,4 \\
\hline 5 & Riesgo de infección $\mathrm{r} / \mathrm{c}$ procedimientos invasivos & 10 & 2,4 \\
\hline 6 & Deterioro de la movilidad física $\mathrm{r} / \mathrm{c}$ debilidad muscular & 9 & 2,1 \\
\hline 7 & Protección ineficaz r/c perfiles hematológicos disminu idos & 8 & 1,9 \\
\hline 8 & Riesgo de deterioro de la integridad cutánea $\mathrm{r} / \mathrm{c}$ inmo vilidad & 7 & 1,7 \\
\hline 10 & Intolerancia a la actividad $\mathrm{r} / \mathrm{c}$ debilidad muscular & 5 & 1,2 \\
\hline 13 & Intolerancia a la activid ad $\mathrm{r} / \mathrm{c}$ fatiga. & 4 & 0,9 \\
\hline \multirow[t]{3}{*}{15} & Déficit de autocuidado: Baño vestimenta alimentación $\mathrm{r} / \mathrm{c}$ deterioro de la percepción cognitiva & 2 & 0,5 \\
\hline & Otros diag nósticos menos frecuentes & 21 & 5,0 \\
\hline & Diagnósticos con factor de riesgo & & \\
\hline 1 & Riesgo de infección $\mathrm{r} / \mathrm{c}$ procedimientos invasivos & 14 & 3,3 \\
\hline 2 & $\begin{array}{l}\text { Riesgo de déficit de volumen de liquido r/c pérdidas excesivas a través de vías normales } \\
\text { (diarrea) }\end{array}$ & 2 & 0,5 \\
\hline \multirow[t]{2}{*}{3} & Riesgo de aspiració n r/c alimentación por so nda naso gástrica & 1 & 0,2 \\
\hline & Total de diagnósticos que cumplen & 187 & 44,1 \\
\hline
\end{tabular}

Tabla 5. Enunciados diagnósticos enfermeros más frecuentes que cumplen con las tres dimensiones según la taxonomía II de NANDA del Servicio de Medicina 4 I del Hospital Nacional Arzobispo Loayza. Julio a septiembre 2011

\begin{tabular}{|c|c|c|c|}
\hline $\mathbf{N}^{\circ}$ & DIAGNÓSTICOS CON LAS TRES DIMENSIONES & $\mathbf{N}$ & $\%$ \\
\hline \multicolumn{4}{|c|}{ Diagnósticos reales } \\
\hline 1 & Dolor agudo r/c agente lesivo biológico & 82 & 19,3 \\
\hline 2 & Ansiedad $\mathrm{r} / \mathrm{c}$ cambios en el estado de salud & 16 & 3,8 \\
\hline 3 & Limpieza ineficaz de las vías aéreas $r$ /c acúmulo de secreciones & 9 & 2,1 \\
\hline 4 & Protección ineficaz r/c perfiles hematológicos disminuidos & 7 & 1,7 \\
\hline 5 & Deterioro de la in tegridad cutánea $\mathrm{r} / \mathrm{c}$ inmovilidad & 6 & 1,4 \\
\hline 6 & Intolerancia a la actividad $\mathrm{r} / \mathrm{c}$ debilidad muscular & 5 & 1,2 \\
\hline 7 & Exceso de volumen de líquidos $\mathrm{r} / \mathrm{c}$ compromiso de los mecanismos reguladores & 5 & 1,2 \\
\hline 8 & Náuseas r/c irritación gástrica & 4 & 0,9 \\
\hline 9 & Deterioro de la movilidad física r/c debilidad muscular & 4 & 0,9 \\
\hline & Déficit de autocuidado: baño, vestimenta, alimen tación $\mathrm{r} / \mathrm{c}$ debilidad muscular & 2 & 0,5 \\
\hline 11 & Deterioro del in tercambio gaseoso $\mathrm{r} / \mathrm{c}$ camb io en la membrana alveo lo capilar & 2 & 0,5 \\
\hline 12 & Estreñimiento $\mathrm{r} / \mathrm{c}$ escasa ingesta de fibra & 1 & 0,2 \\
\hline Total & de diagnósticos que cumplen & 143 & 33,7 \\
\hline
\end{tabular}


NANDA, es de 44,1\%. De los cuales, el más frecuente es el dolor agudo $\mathrm{r} / \mathrm{c}$ agente lesivo biológico con un $15,8 \%$, así como el menos frecuente es el riesgo de aspiración $\mathrm{r} / \mathrm{c}$ alimentación por sonda nasogástrica con un $0,2 \%$.

La Tabla 5 muestra que el total de enunciados diagnósticos enfermeros más frecuentes que cumplen con las tres dimensiones según la Taxonomía II de NANDA es de $33,7 \%$. De los cuales, el diagnóstico real más frecuente es el dolor agudo $\mathrm{r} / \mathrm{c}$ agente lesivo biológico con un $19,3 \%$; así como el menos frecuente es el estreñimiento r/c escasa ingesta de fibra con un $0,2 \%$.

\section{DISCUSIÓN}

Los diagnósticos de enfermería, con el paso del tiempo y el estudio en profundidad de las nuevas tendencias, han sufrido una importante transformación. Actualmente, existen trabajos de colaboración entre enfermeras de distintos países para la difusión, validación y utilización del sistema de clasificación de los diagnósticos de enfermería (5-7). En consecuencia, está teniendo lugar una conjunción de esfuerzos en el nivel internacional que, sin duda, va a tener gran trascendencia para el desarrollo del ejercicio profesional.

En la actualidad el Servicio de Medicina 4I obtiene los datos del paciente mediante la valoración de respuestas humanas, utilizando un formato semiestructurado de notas de enfermería del servicio, donde se encuentran los datos subjetivos, objetivos del paciente, valores de exámenes de laboratorio: hemograma, urea, creatinina y gases arteriales. Además de resultados de procedimientos invasivos y no invasivos: aspirado de medula ósea, paracentesis, ecografia renal, E.C.G; para poder agruparlos como manifestaciones de signos y síntomas que conducen al diagnóstico $(8,17)$.

En el presente estudio los enunciados diagnósticos enfermeros más frecuentes que cumplen con las tres dimensiones según la taxonomía II de NANDA del Servicio de Medicina 4 I del Hospital Nacional Arzobispo Loayza de Lima, registrados por las enfermeras en las notas de enfermería, permite identificar los diagnósticos que utilizan la etiqueta, las características definitorias, el factor relacionado, y el factor de riesgo. Se tuvo como muestra 424 diagnósticos enfermeros, de los cuales 143 (33,7\%) cumplen las tres dimensiones según la Taxonomía II de NANDA, siendo estos el total de diagnósticos aprobados (Tabla 5). Dentro de los diagnósticos aprobados se identificó doce diagnósticos reales, de los cuales los más frecuentes son: 82 diagnósticos de dolor agudo r/c agente lesivo biológico (19,3\%); 16 diagnósticos de ansiedad r/c cambios en el estado de salud $(3,8 \%)$; nueve diagnósticos de limpieza ineficaz de las vías aéreas $\mathrm{r} / \mathrm{c}$ acumulo de secreciones $(2,1 \%)$; siete diagnósticos de protección ineficaz $\mathrm{r} / \mathrm{c}$ perfiles hematológicos disminuidos $(1,7 \%)$; seis diagnósticos de deterioro de la integridad cutánea r/c inmovilidad $(1,4 \%)$; cinco diagnósticos de intolerancia a la actividad $\mathrm{r} / \mathrm{c}$ debilidad muscular $(1,2 \%)$; y cinco diagnósticos de exceso de volumen de líquido r/c compromiso de los mecanismo reguladores $(1,2 \%)$. Tres diagnósticos de riesgo, de los cuales el más frecuente es el de catorce diagnósticos de riesgo de infección $\mathrm{r} / \mathrm{c}$ procedimientos invasivos $(3,2)$. Al analizar la Tabla 5 vemos como diagnostico enfermero más frecuente el dolor agudo $\mathrm{r} / \mathrm{c}$ agente lesivo biológico $(19,3 \%)$ donde se identificó tres características: localización, torácica; tipo, sordo; intensidad, escala de dolor de 3 a 4, siendo un dolor leve a moderado, teniendo como referencia la escala del dolor descriptiva del 1 al 10 según la OMS. El dolor agudo es una experiencia sensitiva y emocional desagradable por una lesión tisular real o potencial con una duración menor de seis meses. Por lo tanto, ocasiona en el paciente trastornos del sueño, gestos de protección, muecas, mirada abatida, y posición antiálgica. El dolor es un mecanismo fisiológico protector; por ejemplo, una persona con el tobillo fracturado evita dejar caer todo su peso sobre el pie enfermo para prevenir un daño mayor. Las personas que padecen enfermedades crónicas presentan el dolor como un síntoma común. El Servicio de Medicina 4 I alberga pacientes con enfermedades crónicas degenerativas como la diabetes mellitus, insuficiencia renal, insuficiencia respiratoria, lupus eritematoso, artritis, osteoporosis, politraumatizados entre otros. Sin embargo, por la complejidad del servicio existen otros diagnósticos enfermeros prioritarios que no se han identificado y registrado por las enfermeras tales como: el desequilibrio nutricional, ingesta inferior a las necesidades; el deterioro de la eliminación urinaria; la disminución del gasto cardiaco; el riesgo de nivel de glicemia inestable; el riesgo de sangrado; el riesgo de shock; la perfusión tisular periférica ineficaz y el riesgo de perfusión renal ineficaz.

Los resultados arriba expuestos presentan alguna similitud con el estudio de Galache (2004), el cual tuvo como objetivo identificar los diagnósticos de enfermería en los pacientes con insuficiencia renal crónica. Se utiliza para la denominación de los diagnósticos, la Taxonomía NANDA II, considerando en cada uno de ellos las características definitorias, los factores relacionados y los factores de riesgo. El resultado del estudio dio un mayor número de diagnósticos de enfermería en los siguientes patrones y por este orden: 
Nutricional-metabólico: destaca el riesgo de infección en los pacientes estudiados (50); exceso de volumen de líquidos en 42 de dichos pacientes; riesgo de deterioro de la integridad cutánea en 25 ; desequilibrio nutricional por defecto en 16; desequilibrio nutricional por exceso en 11, y deterioro de la integridad cutánea en cuatro pacientes. En Actividad-Ejercicio destaca el deterioro en el mantenimiento del hogar en 35 pacientes; la fatiga en 26; el déficit de autocuidado en 15; la intolerancia a la actividad en 12; el déficit de actividades recreativas en 10, y el deterioro de la movilidad física en 10. En AutopercepciónAutoconcepto: el temor, en 43 individuos; la baja autoestima crónica en 15, y los de desesperanza y ansiedad en cinco $\mathrm{y}$ en dos respectivamente. En el patrón cognitivoperceptual, destaca el dolor crónico que se manifestó en 35 pacientes $(9,18-20)$.

Asimismo, según Bertoncello C. y López D. (2007), en el estudio titulado Diagnósticos de enfermería documentados para pacientes de clínica médica, se analizó registros de los diagnósticos de enfermería documentados, tres meses después de la implementación de la clasificación de la NANDA-I. La muestra estuvo constituida por treinta pacientes internados en la clínica médica. Los diagnósticos documentados en el primer día de hospitalización fueron manualmente transcritos y analizados según sus frecuencias. Fueron documentados 144 diagnósticos; los más frecuentes fueron: dolor agudo $(66,7 \%)$; deterioro de la integridad tisular (63,3\%); limpieza inefectiva de las vías aéreas $(43,3 \%)$; riesgo de deterioro de la integridad cutánea $(36,7 \%)$, y deterioro de la integridad cutánea $(33,3 \%)$. Resultados también similares al estudio, los cuales ilustran de alguna manera la escasa identificación de diagnósticos por parte del profesional de enfermería, por lo que solo cubren algunas necesidades básicas del paciente y no su totalidad $(10,21)$.

En el transcurso de la recolección de datos los investigadores identificaron diagnósticos enfermeros más frecuentes que no cumplen con la etiqueta según taxonomía II de NANDA, estos son: diez diagnósticos de dolor leve r/c agente lesivo biológico (2,3\%); ocho diagnósticos de alteración del bienestar físico r/c dolor $(1,8 \%)$; ocho diagnósticos de riesgo de proceso infeccioso $\mathrm{r} / \mathrm{c}$ catéteres invasivos $(1,8 \%)$; ocho diagnósticos de riesgo de lesión caída r/c debilidad muscular (1,8\%); seis diagnósticos de alteración de la deambulación $\mathrm{r} / \mathrm{c}$ debilidad muscular $(1,3 \%)$, y seis diagnósticos de glicemia inestable r/c alteración de los mecanismos reguladores $(1,3 \%)$. También se identificaron diagnósticos enfermeros más frecuentes que no cumplen con las características definitorias según taxonomía II de NANDA: veinte diagnósticos de ansiedad r/c cam- bios en el estado de salud (4,5\%); diecinueve diagnósticos de ansiedad $\mathrm{r} / \mathrm{c}$ estancia hospitalaria (4,3\%); catorce diagnósticos de deterioro de la movilidad física $\mathrm{r} / \mathrm{c}$ debilidad muscular $(3,2 \%)$; doce diagnósticos de riesgo de infección $\mathrm{r} / \mathrm{c}$ catéteres invasivos $(2,7 \%)$; nueve diagnósticos de hipertermia $\mathrm{r} / \mathrm{c}$ proceso infeccioso $(2 \%)$, y ocho diagnósticos de riesgo de lesión caída r/c debilidad muscular $(1,8 \%)$. Se identificaron diagnósticos enfermeros más frecuentes que no cumplen con el factor relacionado y de riesgo según taxonomía II de NANDA, siendo los más frecuentes: veintiún diagnósticos de ansiedad $\mathrm{r} / \mathrm{c}$ estancia hospitalaria $(2,7 \%)$; catorce diagnósticos de hipertermia $\mathrm{r} / \mathrm{c}$ proceso infeccioso $(3,2 \%)$; siete diagnósticos de alteración del bienestar físico $\mathrm{r} / \mathrm{c}$ dolor $(1,6 \%)$, y once diagnósticos de riesgo de infección $\mathrm{r} / \mathrm{c}$ catéteres invasivos (2,5\%). Así mismo, se identificó los diagnósticos más frecuentes que no cumplen la taxonomía II de NANDA, de acuerdo con los tres objetivos específicos de la investigación. Estos resultados son: veinticinco diagnósticos de ansiedad $\mathrm{r} / \mathrm{c}$ estancia hospitalaria $(5,6 \%)$; quince diagnósticos de deterioro de la movilidad física $\mathrm{r} / \mathrm{c}$ debilidad muscular (3,4\%); quince diagnósticos de dolor agudo r/c agente lesivo biológico $(3,4 \%)$; catorce diagnósticos de hipertermia $\mathrm{r} / \mathrm{c}$ proceso infeccioso $(3,1 \%)$; doce diagnósticos de riesgo de infección $\mathrm{r} / \mathrm{c}$ catéter invasivo $(2,7 \%)$; trece diagnósticos de ansiedad $\mathrm{r} / \mathrm{c}$ estado de salud (2,9\%); nueve diagnósticos de riesgo de lesión caída $\mathrm{r} / \mathrm{c}$ debilidad muscular (2\%); otros, 178 diagnósticos (40,4\%), siendo un total de 281 diagnósticos $(66,3 \%)$ de enfermería que no aprobaron.

En los resultados aquí expuestos se pudo observar, mediante la recolección de los diagnósticos enfermeros, diversos errores ortográficos y la transcripción errónea e incompleta de la etiqueta diagnostica; características definitorias que no agrupan los signos y síntomas del problema de salud de cada paciente, debido a la dificultad de la enfermera para identificarlos y, en algunos casos, no se evidencio en los datos subjetivos y objetivos. En el factor relacionado y de riesgo no se identifico los factores causantes o que contribuyen a la respuesta manifestada por el paciente. Este $66,3 \%$ de diagnósticos enfermeros no aprobados se debería a que algunas enfermeras han sido incorporadas al servicio de medicina 4I recientemente. En otros casos es debido al tiempo que le toma a la enfermera planificar las actividades de enfermería sin poder realizar las notas de enfermería de manera eficaz e eficiente; así como la falta de actualización de los diagnósticos enfermeros debido a que no se capacitan en la actualidad.

En los resultados de la Tabla 3 se encontró que del total 
de diagnósticos enfermeros evaluados y registrados, 147 diagnósticos $(34,7 \%)$ cumplen con las características definitorias según la Taxonomía II de NANDA siendo las más frecuentes el dolor torácico, tipo sordo, escala del dolor 3 o 4; el nerviosismo, la preocupación, los movimientos extraños, la estancia prolongada de vía periférica, la estancia prolongada de SNG, el cambio de $\mathrm{pH}$ de las secreciones, el calor al tacto, la taquipnea, la piel enrojecida, la tos inefectiva, la excesiva cantidad de esputo, la agitación, entre otros.

Asimismo, en la Tabla 4, 187 diagnósticos $(44,1 \%)$ cumplen con el factor relacionado según la taxonomía II de NANDA, muestran el tipo de relación con el diagnóstico, pudiendo ser fisiopatológicos, psicológicos, genéticos y ambientales; están relacionados con el tratamiento, y los factores de situación y maduración, los que pueden influir en el estado de salud o en el desarrollo del problema (8, $11,22,23)$.

Fujishima J y col en su estudio cuyo objetivo fue identificar los diagnósticos de enfermería de pacientes ingresados en la Unidad de Enfermedades Infecciosas y Parasitarias en un hospital universitario, y examinar la denominación, los factores relacionados y las características que definen los diagnósticos, y su adecuación a la nomenclatura de la taxonomía NANDA 2008; como resultado, encontraron 105 diagnósticos reales y 18 de riesgo. Doce diagnósticos $(10 \%)$ correspondían totalmente a la taxonomía. Las denominaciones más frecuentes fueron: «Riesgo de infección» (100\%) y «Ansiedad» (32\%). En los diagnósticos reales las denominaciones, los factores relacionados y las características definitorias estaban de acuerdo con la taxonomía en 76,27 y $23 \%$, respectivamente. En los diagnósticos de riesgo las denominaciones y los factores de riesgo fueron adecuados en 83 y $72 \%$. La principal dificultad encontrada en este estudio fue la identificación de los enunciados diagnósticos enfermeros que cumplían con los factores relacionados, y de las características definitorias $(12,24)$.

Estudios realizados por enfermeros evidencian el interés por validar, con poblaciones en diferentes estados de salud, las etiquetas de los diagnósticos de enfermería y sus respectivas características definitorias. Entre estos se puede citar a Quatrini Carvalho, H. Leite de Barrios Alba Lucia y Rivero de Gutiérrez, Maria Gaby (2000), quienes llevaron a cabo el estudio «Identificación de las características definitorias del diagnóstico de enfermería exceso del volumen de líquidos», que fue realizado con 29 pacientes; los resultados mostraron que las dos enfermeras evaluadoras concordaron en la indicación de presencia de las características del diagnóstico objeto de estudio en nueve pacientes, y de ausencia en veinte (13). Al evaluar los resultados se encontró en el diagnóstico un exceso de volumen de líquidos, que nueve cumplen con la etiqueta y definición NANDA, pero solo dos cumplen con las características definitorias y el factor relacionado. En suma, en enfermería los diagnósticos representan una herramienta para normalizar el lenguaje entre enfermeros y mejorar la calidad de la atención; sin embargo, es evidente que en nuestra realidad la falta de preparación profesional y profundización en su comprensión hacen limitado su empleo. La elección de los resultados e indicadores para guiar la propuesta de intervenciones de enfermería depende de las habilidades clínicas de las enfermeras, especialmente de aquellos involucrados en el diagnóstico de la enfermedad. Un diagnóstico de enfermería puede ser más o menos preciso. Un diagnóstico poco preciso puede conducir a la ejecución de intervenciones innecesarias e incluso perjudiciales para el paciente, deteriorando la calidad de la atención. Por lo tanto, la preocupación por la exactitud de los diagnósticos establecidos por las enfermeras debe estar entre las prioridades de los servicios de enfermería $(4,25)$.

Se trata entonces de contribuir a la actualización del conocimiento del profesional de enfermería sobre la clasificación y estructura de los diagnósticos NANDA, que continua cada año haciendo revisiones y actualizaciones de la taxonomía y poniéndola a disposición de todos los profesionales de Enfermería en el mundo, para dirigir la estandarización de lenguaje de los diagnósticos de enfermería, lo que implica una continua capacitación para el desarrollo de esta área. Estudios sobre la estructura de la taxonomía II ayudarán a promover la calidad de los cuidados de enfermería. Es trabajo difícil, pero con la práctica y la experiencia se mejorará las habilidades y competencias tanto intelectuales y técnicas como interpersonales, permitiendo así identificar y estructurar adecuados diagnósticos que serán la base para las intervenciones de enfermería en el cuidado del paciente.

\section{REFERENCIAS BIBLIOGRÁFICAS}

1. Rodrigo M, Fernández C, Navarro M. De la teoría a la practica: el pensamiento de Virginia Henderson en el siglo XXI.3ra ed. España: Elsevier Masson; 2009.

2. Cisneros F. Enfermera especialista proceso de atención de Enfermería. Madrid. 2008. [Internet] [citado el 09 de julio del 2009] Disponible en: http:// atenea.unicauca.edu.co/.../ProcesoDeAtencion DeEnfermeria-PAE 
3. Iyer P, Taptich B. Proceso y Diagnósticos de Enfermería. 3 edición. México. D. F: McGraw-Hill; Interamericana. 1997.

4. Carrillo G. Rubiano M. La Investigación en validación de diagnósticos de enfermería. Rev. Cubana Enfermera; 2007. [Internet] [citado el 12 de agosto del 2010]; 23(3): Disponible en: http://bvs.sld.cu/ revistas/enf/vol23 3 07/enf09307.html

5. Quatrini Carvalho H, Leite de Barrios A, Gutierrez $\mathrm{M}$, Identificación de las caracteristicas definitorias del diagnóstico de enfermería exceso de volumen de liquidos. [Internet] [citado el 10 de agosto del 2011].Disponible en:http://www.scielo.br/scielo.php? script=sci_abstract\&pid=S010411692000000200011 $\& \operatorname{lng}=$ en\&nrm=iso \&tlng $=$ es

6. Alfambra M. I, Alonso D. M, Rodríguez M. A, Ramos C. E. Informe de alta de enfermería: un instrumento para la continuidad de los cuidados. Index de Enfermería 1996. [Internet] [citado el 09 de julio del 2009]. Disponible en: http://europa.sim.ucm.es/ compludoc.

7. Wells M. Planificación de altas. Cómo llenar los vacíos de una atención continuada. Nursing de enfermería. 1984; [Internet] [citado el 09 de julio del 2009]. Disponible en: http://www.index-f.com/lascasas/ documentos/lc0012.php.

8. NANDA Internacional; Diagnósticos Enfermeros: Definiciones y Clasificación 2009 - 2011. Tercera Edición. España: Editorial Elsevier; 2003.

9. Galache A. Diagnósticos de enfermería en pacientes con insuficiencia renal crónica. Rev. Cubana enfermera; 2009. [Internet] [Citado el 10 de agosto del 2010]; 23(1): Disponible en: http://bvs.sld.cu/ revistas/enf/vol23 1 07/enf09307.html

10. Bertoncello C. Diagnósticos de enfermería documentados para pacientes de clínica médica. [Internet] [citado el 10 de agosto del 2011]. Disponible en: http://www.scielo.br/pdf/reeusp/v41n3/08.pdf

11. Fernández F. Modelos y teorías de enfermería. Breve historia de la ciencia enfermera. En: Enfermería fundamental. Barcelona: Masson; 1995. [Internet] [citado el 09 de julio del 2009]. Disponible en: http:// www.indexf.com/lascasas/documentos/1c0012.php.

12. Fujishima J. Consorti F. Cristiano T. Ceolim M. Diagnósticos de enfermería de pacientes ingresados con Enfermedades Infecciosas. [Internet] [citado el 10 de agosto del 2011]. Disponible en: http:// www.scielo.br/scielo.php?pid=S010321002010000600016 \&script $=$ sci abstract\&tlng=es

13. Consejo General de Enfermería; Sistema de Clasificación; 2009. [Internet] [citado el 13 de mayo del 2009] Disponible en: http://www.nipe.isics.es/ servlet.

14. Ibarra A, Diagnósticos de Enfermería (NANDA) Aplicados A Los Cuidados Críticos Pediátricos Y Neonatales. Madrid. 2000. [Internet] [actualizado 2010 diciembre 06; citado el 12 de marzo del 2010]. Disponible en: http://www.eccpn.aiba rra.org/temario/ seccion1/capitulo22/capitulo22.htm

15. Rodríguez S. El lenguaje común enfermero y la normalización de la práctica: un camino hacia la excelencia enfermera [página web en internet] Madrid. 2004. [Internet] [actualizado 2009 noviembre 12; citado el 12 de marzo del 2010] Disponible en: http://www.ee.isics.es/servlet/Satellite? pagena $\mathrm{me}=$ ExcelenciaEnfermera/Articulo_EE/planti 1la_articulo_EE\&numRevista $=0 \&$ id Articulo= 1082026113311

16. Gutiérrez O. Factores que determinan la aplicación del proceso de enfermería en instituciones hospitalarias de Villavicencio. Colombia: ISSN, 2004. [Internet] [actulizado 2008 noviembre 20; citado el 12 de marzo del 2010] Disponible en: http://www. lalibreriadelau. $\mathrm{com} / \mathrm{lu} /$ affiliates 2. php? affiliate $=17 \&$ to $=$ product $\&$ products_id $=35029$

17. Mompart G, Durán E. M. Utilización del diagnóstico enfermero en los informes de enfermería. Biblioteca Lascasas, 2005. [Internet] [citado el 09 de julio del 2009]. Disponible en: http://www.index-f.com/ lascasas/documentos/lc0012.php.

18. Camacho O, Llanes M, García R, Romero C. Informe de enfermería al alta. Rol de enfermería. 1998. [Internet] [citado el 09 de julio del 2009] Disponible en: http://www.doyma.es/revistas/ctl_servlet

19. Torres N, Cazorla P, Fernández A, Fernández L, Gil M. ¿Qué opinan los enfermeros de atención primaria de los informes de enfermería al alta hospitalaria? Enfermería. Científica 2000; [Internet] [citado el 09 de julio del 2009]. Disponible en: http://www.ucm.es/ BUCM/compludoc/S.htm

20. Avalos T, Silé R, Diagnostico de Enfermería. Evolución histórica y diagnósticos más frecuentes en afecciones cardiovasculares. [Internet] [Citado el 20 de marzo del 2010] Disponible en: Clarahttp:// www.portalesmedicos.com/publicaciones/articles/ 838/1/ Diagnostico-de-Enfermeria-Evolucionhistorica-y-diagnosticos-mas-frecuentes-enafecciones -cardiovasculares.html

21. Carpenito L; Diagnostico de enfermería. Aplicación en la práctica clínica. Editorial interamericana de España, 3ra edición. 1991.

22. Carvalho. M, Lopes S, Cavalcanti G, Machado T, Marinho M. Diagnósticos de enfermería como 
instrumentos en la formación del enfermero: una revisión de la literatura [citado el 20 de marzo del 2010] Disponible en: http://scielo.isciii .es/ scielo.php?pid=S1695-61412009000300020\& script $=$ sci_arttext

23. Bes G. La aplicación práctica a través del autocuidado. Index Enfermería. 1995 [Citado el 09 de julio del 2009].Disponible en: http://europa.sim.ucm.es/ compludoc.

24. Ferreiro C. Consideraciones sobre la ética y perfil profesional. Cuaderno de Bioética. 1998.

25. Código Colombiano de Ética en Enfermería. Capitulo 2. Principios de Ética. 2002. [Citado el 09 de julio del 2009].Disponible en: http://www.r-e-a-l.org/subredescondetica-cap02.

\section{Correspondencia}

Peralta Julio

Jr. Carlos de los Heros 121 - 25, Pueblo Libre. Lima 21

Correo electrónico: julio.peralta@upch.pe

Forma de citar este artículo: Peralta Julio, Saravia Lizbbet. Enunciados diagnósticos enfermeros más frecuentes, que cumplen con la taxonomía II NANDA registrado en el servicio de medicina del Hospital Nacional Arzobispo Loayza. Rev enferm Herediana. 2012;5(2):78-88. 